\title{
Automated Diagnose of Neovascular Glaucoma Disease using advance Image Analysis Technique
}

\author{
Pavitra G. \\ Dept. of ECE \\ HKBKCE. Bangalore
}

\author{
T.C. Manjunath \\ Dept. of ECE \\ HKBKCE. Bangalore
}

\author{
Dharmanna \\ Lamani \\ Dept. of CSE \\ SDMIT, Ujire
}

\author{
Chandrappa S. \\ Dept. of CSE \\ SDMIT, Ujire
}

\begin{abstract}
Neovascular glaucoma is a secondary glaucoma that is developed due to improper control of glucose level in diabetic patients. This disease is highly complex to manage and can lead to permanent vision loss. Once this disease develops, early detection plays very important role to ophthalmologist to start treatment in order to reduce the visionlossprogression. Current detection techniques detect the glaucoma after $40 \%$ vision loss. In this research paper, the authors are presenting diagnosis of neovascular glaucoma using image feature fractal dimension. Fractal dimension is a unique parameter to identify healthy and neovascular retina. In this work, we are adapted Support vector machine for the classification of result. Result of our proposed method shows that Fractal dimension values found for the healthy retina is in the range of 1.20 to 1.70 , whereas for neovascular glaucoma affected retina, it exceeds the upper limit.
\end{abstract}

\section{General Terms}

Neovascular Glaucoma, Retina

\section{Keywords}

Fractal Dimension, Blood Vessels, Box Counting

\section{INTRODUCTION}

Neovascular Glaucoma (NVG), the word literally means building new vessels which causes ocular disorders. NVG is also called as Secondary Glaucomaand it is the severe state of glaucoma which may lead to permanent loss of vision if not treated in early stage.

The term neovascular glaucoma was proposed by Weiss in 1963. Earlier it was named as rubeotic, congestive and hemorrhagic glaucoma. This is caused due to the insufficient pumping of oxygen and glucose to the retina. As the result, body starts building new blood vessels in wrong places to keep the tissues alive. These new blood vessels block the aqueous outflow and these new blood vessels are accompanied by fibrous membrane result in peripheral anterior synechiae and progressive angle closure. This increases the intraocular pressure in eyes which may lead to blindness. The ocular diseases like neovascularization of iris (NVI) or neovascularization of angle may finally lead to neovascular glaucoma.

Unfortunately, neovascularization mainly affects small vessels. Detection of these small vessels is very difficult. Also, this technique was more laborious and error prone. Hence, in this paper we presented an appropriate method to detect small vessels which may lead to glaucoma. In Figure 1, left image shows fundus image of healthy eye and right image shows the retina affected by neovascular glaucoma. This paper is organized by following sections. Section 1 presents the introduction of the neovascular glaucoma and its causes. Section 2 describes methods, such as blood vessels extraction, masking boundary of the retinal image and box counting fractal dimension procedure, used for estimation of fractal dimension. Section 3 reveals the result and discussion of present work. Section 4 presents the conclusion of research work followed by references.

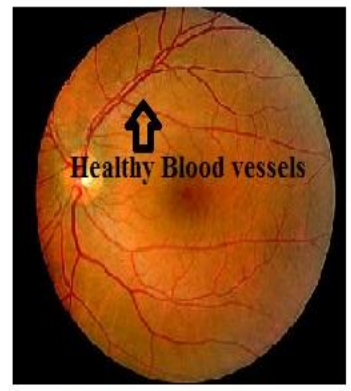

(a) Healthy Retina

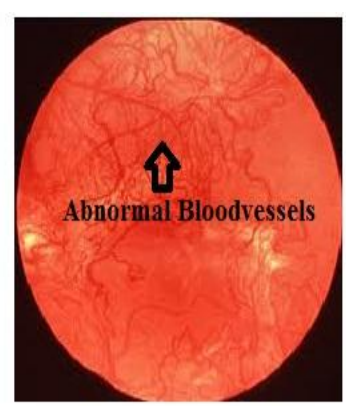

(b) Neovascular Retina
Figure 1: Fundus images (a) Healthy retina and (b) Neovascular retina

\section{METHODS}

This section describes the methods adopted to extract blood vessels and image features using fractal dimension in the A and $\mathrm{B}$ section respectivelly.

\subsection{Blood vessels extraction}

Kirsch templates of size $3 \times 3$ are adopted for the segmentation of Blood Vessels (BV) from fundus retinal digital image. Edge recognition is a process of categorizing the pixel values in order to obtain regular and abrupt changes. Usually result of edge recognition using Kirsch template is used to generate an image containing gray level pixels of value in between 0 to 255 . The value 0 of pixel gray signifies a black pixel and the value 255 signifies a white pixel. Edge information of a particular and target pixel is verified by estimating the brightness level of the adjacent pixels. If there is no major difference in the adjacent pixel brightness levels then there is no option of edge in the retinal image. The depicted methods are the most common and fundamental approaches among all the available edge detection algorithms such as, Prewitt, Canny, Sobel etc. In this work Kirsch template method is adopted for the segmentation of $\mathrm{BV}$ from digital retinal fundus images. The Kirsch edge identificationmethod applies a single mask of size $3 \times 3$ and rotates it in 45 degree increments through all eight directions as shown in Figure 2. 


$$
\begin{aligned}
& {\left[\begin{array}{lll}
-3 & -3 & 5 \\
-3 & 0 & 5 \\
-3 & -3 & 5
\end{array}\right]\left[\begin{array}{lll}
-3 & 5 & 5 \\
-3 & -3 & 5 \\
-3 & -3 & -3
\end{array}\right]\left[\begin{array}{lll}
5 & 5 & 5 \\
-3 & 0 & -3 \\
3 & -3 & -3
\end{array}\right]\left[\begin{array}{lll}
5 & 5 & 3 \\
5 & 0 & -3 \\
-3 & -3 & -3
\end{array}\right]} \\
& {\left[\begin{array}{lll}
5 & -3 & -3 \\
5 & 0 & -3 \\
5 & -3 & -3
\end{array}\right]\left[\begin{array}{lll}
-3 & -3 & -3 \\
5 & 0 & -3 \\
5 & 5 & -3
\end{array}\right]\left[\begin{array}{lll}
-3 & -3 & -3 \\
-3 & 0 & -3 \\
5 & 5 & 5
\end{array}\right]\left[\begin{array}{lll}
-3 & -3 & 5 \\
-3 & 0 & 5 \\
-3 & 5 & 5
\end{array}\right]}
\end{aligned}
$$

Figure 2: Kirsch template

The edge level of the Kirsch operator is estimated as the outmost level across all direction. The matrix includes the information of a pixel and it'sadjacent. The Kirsch method distinguishes direction of the edge as well as an edge. Therefore, there are 8 possible directions such as south, east, north, west, northeast, southeast, southwest and northwest as shown in Figure 2. Out of the various templates, the prime one is considered for the output value and later the edges are segmented. Kirsch template can set and reset the threshold values to obtain most suitable edge of images. Kirsch template also works fit for images having obvious difference between the foreground and background. Since the retinal blood vessels can be considered as diagnostic parameter in fundus retinal digital images. Therefore, for the extraction of Kirsch method is efficiently adopted.

\subsection{Fractal Dimension}

Fractal dimension is a statistical quantity that indicates how completely geometric figure (each part of which as the same statistical character as the whole) fills the space when viewed at finer scales. The fractal dimension is given by the formula

$$
D=\lim _{r \rightarrow 0} \frac{\log N_{r}}{\log \left(\frac{1}{r}\right)}
$$

It is an effective measure to extract features of complex objects and surfaces such as coastlines, mountains, clouds and texture.

Many methods have been developed for estimating fractal dimension. Typically we are considering two methods

- Spectral analysis

- Box counting

Spectral analysis: it is generally implemented as fast Fourier transformation to image and obtains the coefficient and mean of the spectral energy density. By using spectral analysis the fractal dimension can be estimated by analyzing the power law dependency of spectral energy density and square size.

Box counting: It is a method of gathering data for analyzing complex patterns by breaking a dataset, object, image, etc. into smaller and smaller pieces, typically "box"shaped, and analyzing the pieces at each smaller scale. Box counting is the most frequently used method for determining fractal dimension. The objects are partitioned into boxes of certain size. The ratio $r$ in equation is to decide the box size. The task of box counting method is to count the total number of boxes that are needed to form the object. The fractal dimension of the equation can be estimated from the least square linear fit of $\log \mathrm{N}_{\mathrm{r}}$ versus $\log \frac{1}{\mathrm{r}}$. Here, the counting $\mathrm{N}_{\mathrm{r}}$ is for different scaling ratio $\mathrm{r}$. In box counting algorithm, the database or images are first zoomed in or out using optical or computer based method to examine how observations of the images changes with scale. Initially we assigned it with different scale values for datasets or images. In this method, instead of changing the magnification or resolution of a lens the algorithm changes the size of the element used to inspect the object. Figure 3 describes the feature extraction from healthy and unhealthy retinal images. In step 1 color fundus image is given as input to the software later converted into grayscale image. In step 2 boundary of retinal image is masked. In step 3 the grayscale image is converted into binary image using MATLAB im2bw function. In step 4 the fractal dimension feature are extractedusing box counting algorithm.

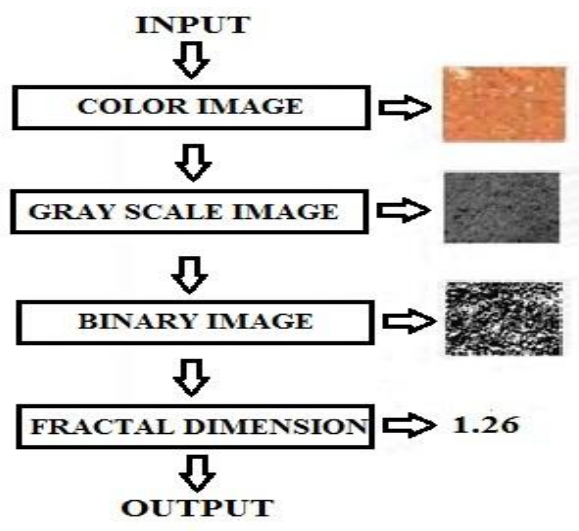

Figure 3: Data flow diagram of proposed method

\section{RESULT AND DISCUSSION}

This section describes the simulation obtained from the healthy retinal fundus images and neovascular fundus retinal images. Fractal dimension and retinal blood vessels extraction algorithms were implemented in MATLAB_13 software. Digital retinal imaging play more and more outstanding role in the identification and treatment of an eye diseases and obtaining of clinically helpful information has become central task. The retinal vasculature analysis using fractal dimension method helps to define the characteristics and extent of diseases like diabetic retinopathy, glaucoma, neovascular glaucoma etc. That will assist to detect and providetreatment for the disease. Consequently, extraction of these features from the retinal fundus images becomes a key challenge for proper analysis, visualization and quantitative comparison. This has been the main focus of this paper that is extraction of blood vessels from digital fundus retinal images. In this work 25 healthy digital retinal images and 25 neovascular retinal images are employed to estimate the method. Some of the images were discarded by ophthalmologists prior to the diagnosis. These images were included in the database to verify the robustness of the developed technique. Image preprocessing was performed in order to enhance the quality of retinal images before subjected to extraction of the blood vessels. After the enhancement of the color retinal image, they are converted into grayscale image using rgb2gray scale image function. By using kirsch template algorithm it segments the blood vessels in the retinal image. For each image in the database fundus mask was detected, that facilitated the detection of vessel pixels within the region of 
interest. The colored healthy fundus retinal images are shown in Figure 4 (a) and all retinal images converted into gray scaled images as shown in Figure4 (b), then gray scaled images are processed by kirsch templates and the segmented blood vessels through edge detection technique is shown in Figure 4(c) and the Figure 4(d) describes outer boundary of retinal image masked and retained blood vessels for the analysis, Figure 4(e) shows the estimated fractal dimension i.e., $\mathrm{FD}=1.6233$. The colored neovascular glaucoma affected fundus retinal images are input to algorithm as shown in Figure 5(a) and all retinal images converted into gray scaled images are shown in Figure 5(b), then gray scaled images processed by kirsch's templates and the segmented blood vessels through edge detection technique are shown in Figure 5(c) and Figure 5(d) describes outer boundary of retinal image

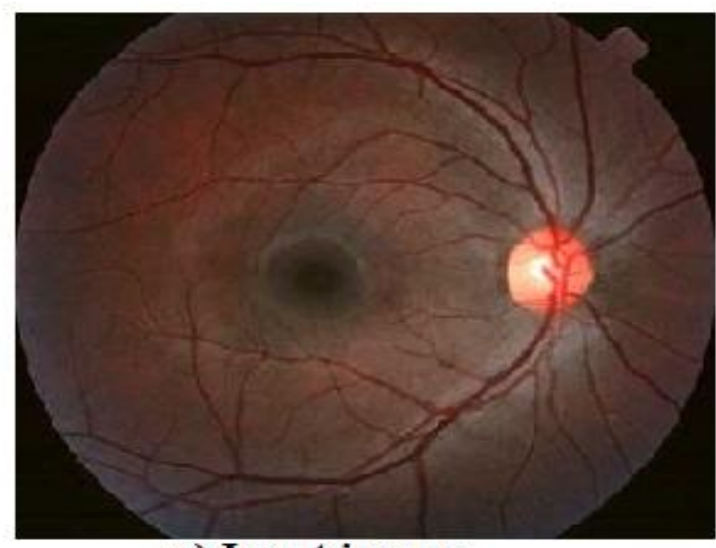

a) Input image

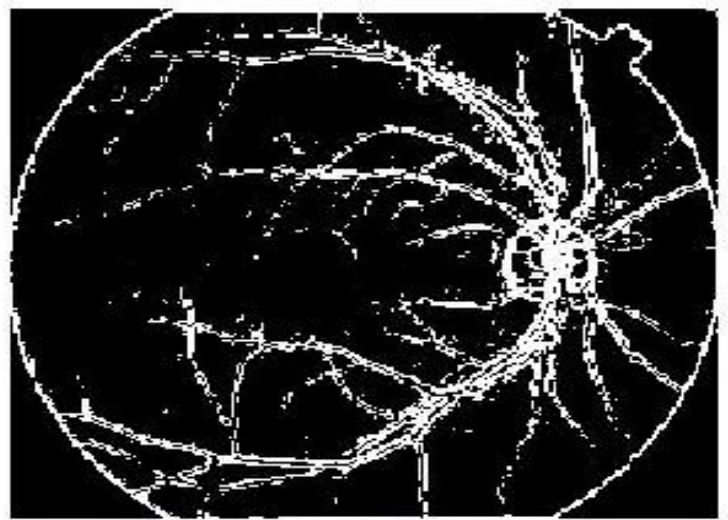

c) Blood vessels Extraction masked and retained only blood vessels for the analysis, Figure 5(e) shows the estimated fractal dimension i.e., FD $=1.9345$. Figure 6 represents the fractal dimension values of healthy retinas (Blue stars) and neovascular retinas (red circles). It is observed that healthy retinal fractal dimension in the range of 1.20 to 1.70 , whereas for neovascular retina above 1.70 to 1.99 , because neovascular retina has higher rough surface due to abnormal growth of blood vessels. Hence fractal dimension of unhealthy retina (neovascular retina) is higher as compared to healthy retina. This relates concept of fractal dimension theory. Table 1 shows the fractal dimension values of healthy and unhealthy retinas for 25 images each. Table 2 depicts first order statistical data like min, max, mean, range, mode, median, standard deviation etc. for healthy and unhealthy retinal eye images.

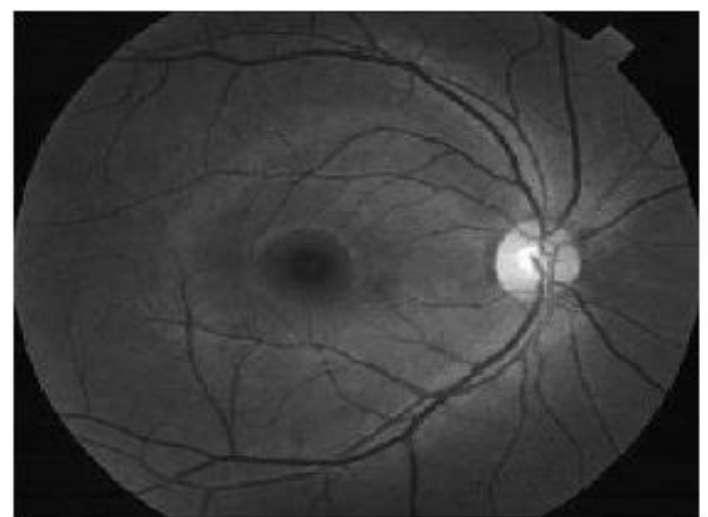

b) Gray Scale image

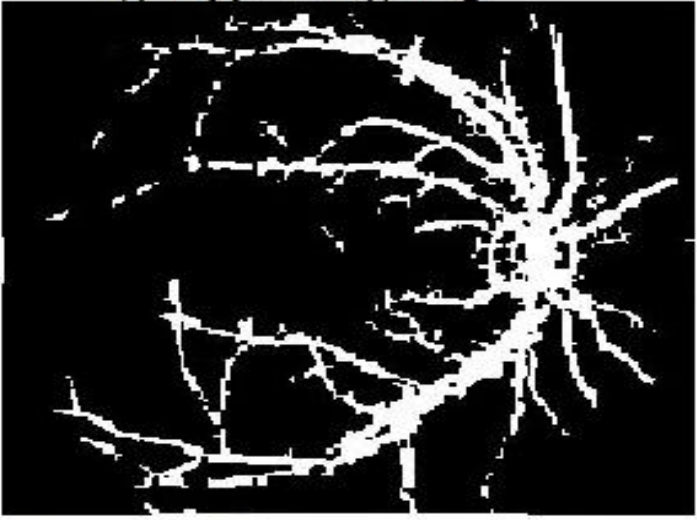

d) Removed perimeter of retina

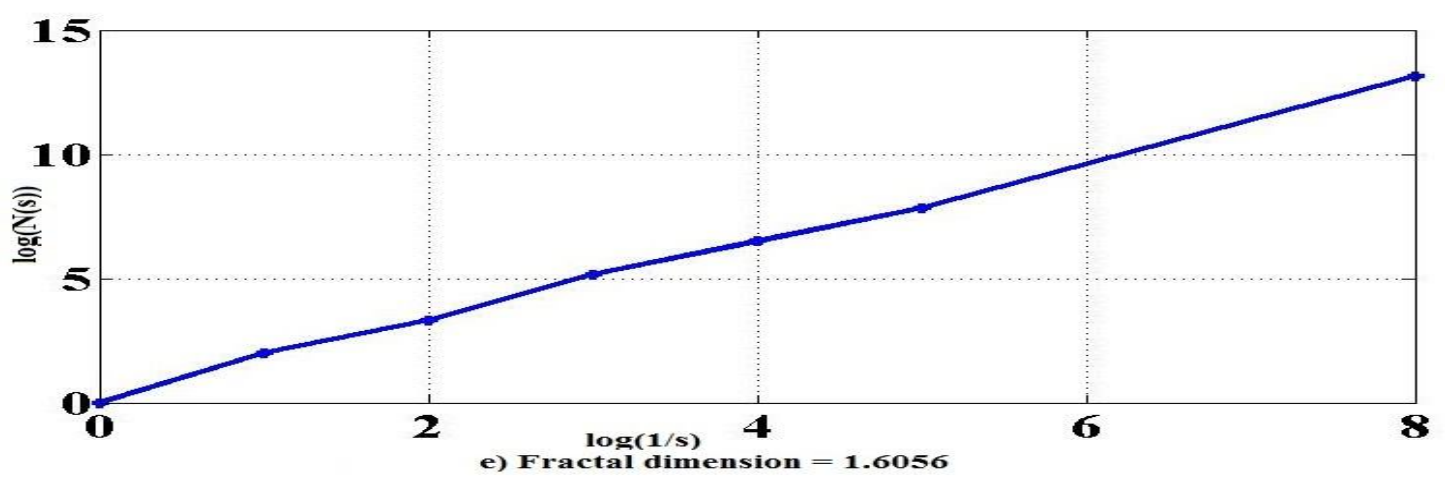

Figure.4: Simulation Result of healthy retina 


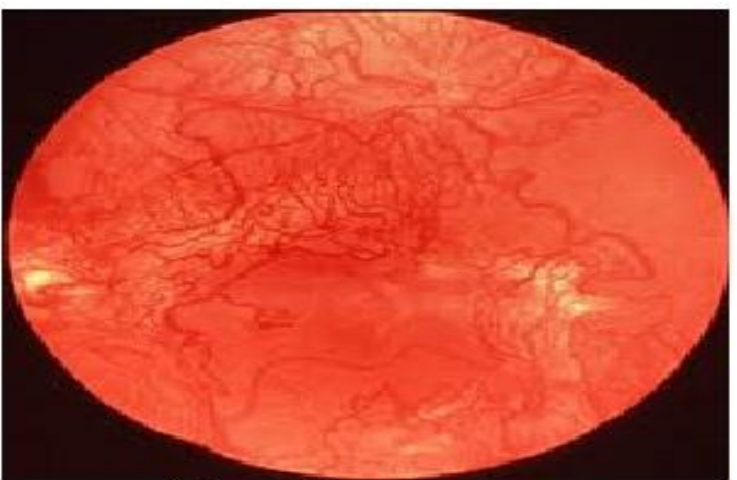

a) Input image

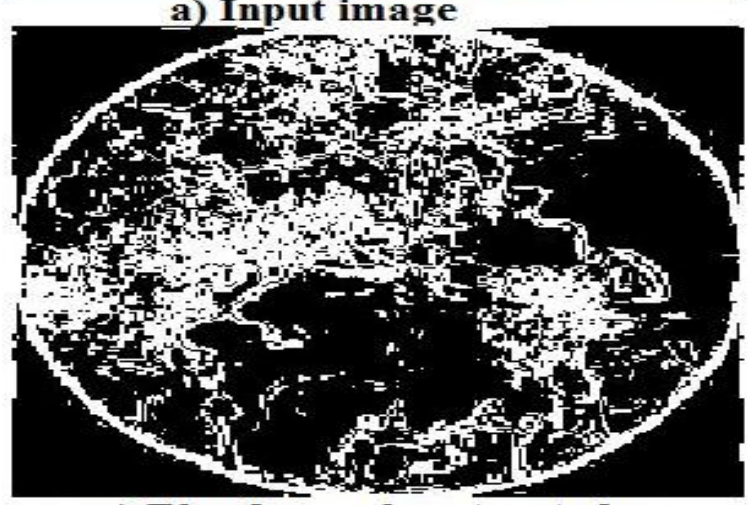

c) Blood vessels extracted

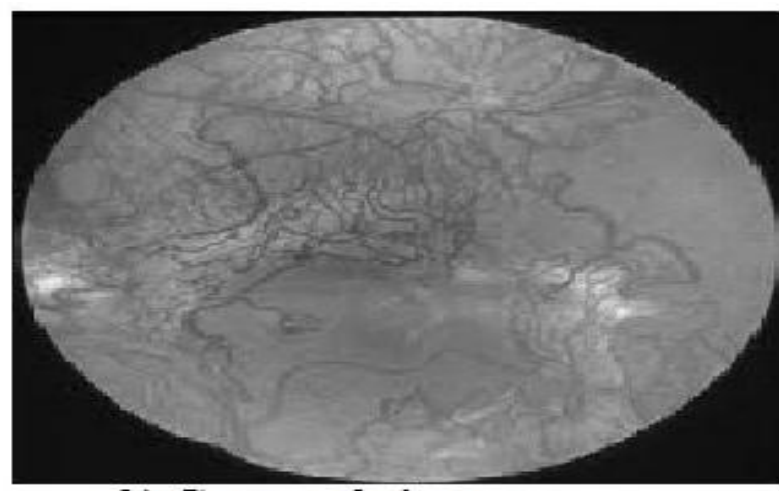

b) Gray scale image

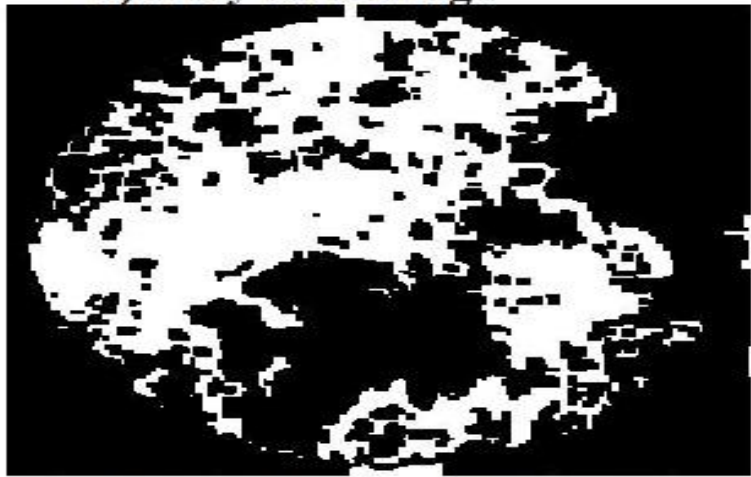

d) Removed outer boundary

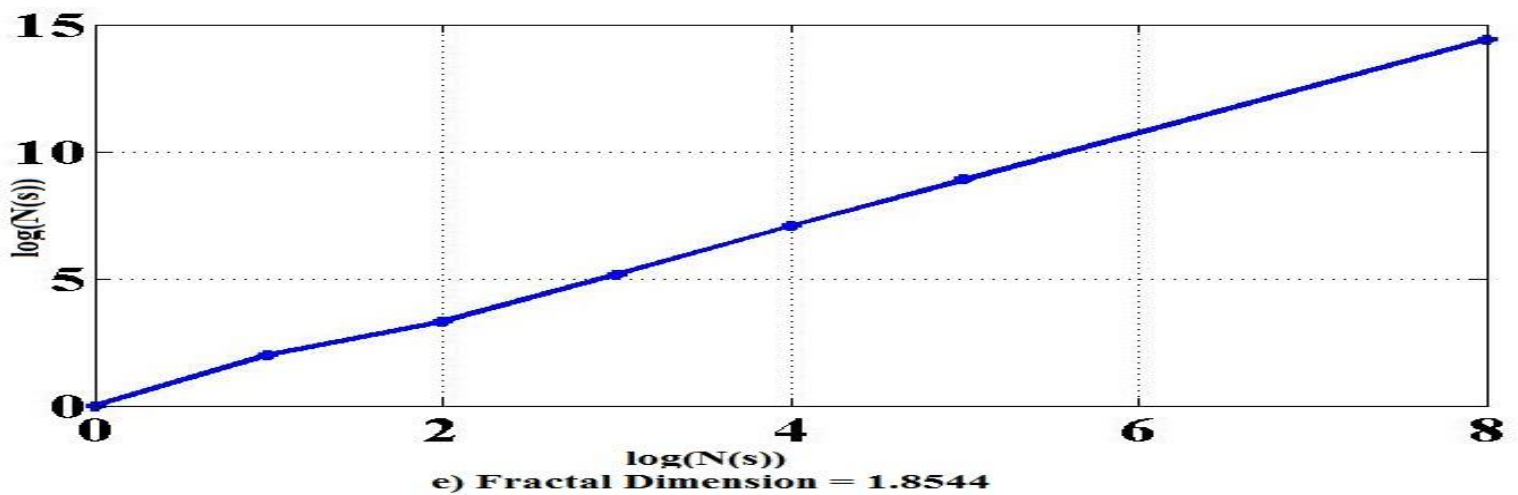

Figure 5: Simulation Result of Neovascular Glaucoma

Table 1: Fractal dimension Values of Healthy and neovascular retinal images

\begin{tabular}{|c|c|c|c|c|}
\hline SL. No & Health Images & FD & Neovascular Image & FD \\
\hline 01 & Healthy_00001 & 1.22 & Neovascular_00001 & 1.71 \\
\hline 02 & Healthy_00002 & 1.56 & Neovascular_00002 & 1.82 \\
\hline 03 & Healthy_00003 & 1.67 & Neovascular_00003 & 1.95 \\
\hline 04 & Healthy_00004 & 1.52 & Neovascular_00004 & 1.86 \\
\hline 05 & Healthy_00005 & 1.59 & Neovascular_00005 & 1.83 \\
\hline 06 & Healthy_00006 & 1.67 & Neovascular_00006 & 1.75 \\
\hline
\end{tabular}




\begin{tabular}{|c|c|c|c|c|}
\hline 08 & Healthy_00008 & 1.29 & Neovascular_00008 & 1.74 \\
\hline 09 & Healthy_00009 & 1.27 & Neovascular_00009 & 1.97 \\
\hline 10 & Healthy_00010 & 1.61 & Neovascular_00010 & 1.86 \\
\hline 11 & Healthy_00011 & 1.25 & Neovascular_00011 & 1.79 \\
\hline 12 & Healthy_00012 & 1.33 & Neovascular_00012 & 1.77 \\
\hline 13 & Healthy_00013 & 1.43 & Neovascular_00013 & 1.84 \\
\hline 14 & Healthy_00014 & 1.27 & Neovascular_00014 & 1.81 \\
\hline 15 & Healthy_00015 & 1.58 & Neovascular_00015 & 1.85 \\
\hline 16 & Healthy_00016 & 1.42 & Neovascular_00016 & 1.76 \\
\hline 17 & Healthy_00017 & 1.47 & Neovascular_00017 & 1.91 \\
\hline 18 & Healthy_00018 & 1.46 & Neovascular_00018 & 1.88 \\
\hline 19 & Healthy_00019 & 1.68 & Neovascular_00019 & 1.70 \\
\hline 20 & Healthy_00020 & 1.17 & Neovascular_00020 & 1.73 \\
\hline 21 & Healthy_00021 & 1.50 & Neovascular_00021 & 1.84 \\
\hline 22 & Healthy_00022 & 1.53 & Neovascular_00022 & 1.93 \\
\hline 23 & Healthy_00023 & 1.53 & Neovascular_00023 & 1.90 \\
\hline 24 & Healthy_00024 & 1.57 & Neovascular_00024 & 1.73 \\
\hline 25 & Healthy_00025 & 1.39 & Neovascular_00025 & 1.81 \\
\hline
\end{tabular}

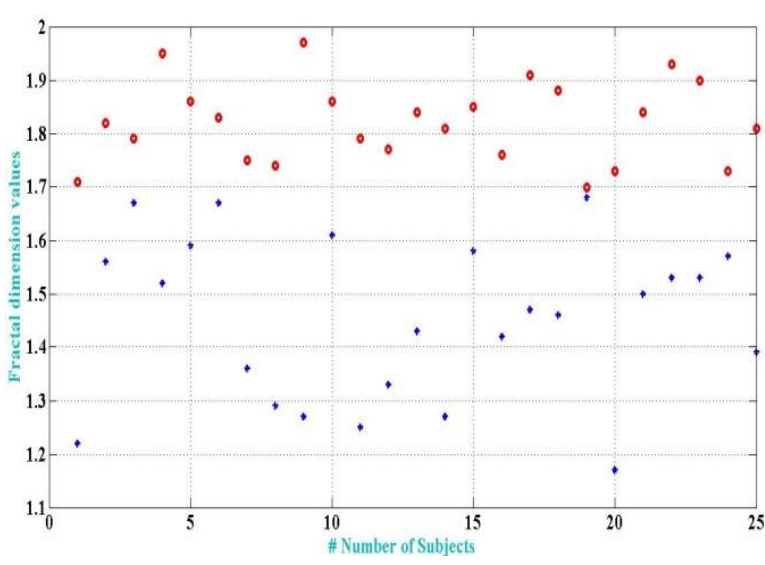

Figure 6: Graph of healthy and neovascular glaucoma FD values

Table 2: Statistical Result of Healthy and Neovascular Retina

\begin{tabular}{|c|c|c|c|}
\hline SL.No & Features & $\begin{array}{c}\text { Healthy } \\
\text { Retina }\end{array}$ & $\begin{array}{c}\text { Neovascular } \\
\text { Retina }\end{array}$ \\
\hline 1 & Min & 1.17 & 1.70 \\
\hline
\end{tabular}

\begin{tabular}{|c|c|c|c|}
\hline 2 & Max & 1.68 & 1.97 \\
\hline 3 & Mean & 1.45 & 1.82 \\
\hline 4 & Median & 1.47 & 1.82 \\
\hline 5 & Mode & 1.27 & 1.73 \\
\hline 6 & SD & 0.15 & 0.07 \\
\hline 7 & Range & 0.51 & 0.27 \\
\hline
\end{tabular}

\section{CONCLUSION}

Detection of neovascular glaucoma still remains as a challenging task, despite of much advancement in the treatment. Early detection of neovascularization and providing adequate treatment may prevent a visual loss outcome of this disease. Once intraocular pressure increases, successful management of the disease may be very complex. Hence neovascular glaucoma detection in the early stage using proposed image analysis technique paly the major role in prevention of vision loss. Here, Blood vessels extracted from the fundus retinal images are then adapted by box counting fractal dimension method for extraction of image feature. These feature fed to SVM for classification of healthy and unhealthy retina. This method is automatic and loss effective 
compared to other methods. Also, this method can be adapted in public place in order to increase the diagnosis rate. Further analysis of retinal image using different fractal dimension technique left as a future work.

\section{REFERENCES}

[1] K.Narasimhan and Dr.K.Vijayarekha,"An efficient automated system for glaucoma detection using fundus images", Journal of Theoretical and Applied InformationTechnology, ISSN: 1992_8645, Vol.33, No.1, pp.104_110, November 2011.

[2] ArulmozhivarmanPachiyappan, Undurti N Das, Tatavarti VSP Murthy and RaoTatavarti," Automated diagnosis of diabetic retinopathy and glaucoma using fundus and OCT images", Pachiyappan et al. Lipids in Health and Disease, ISSN.11_73,pp.1_10,June 2012.

[3] Rüdiger Bock, Jörg Meier, László G, Nyúl, Joachim Hornegger, Georg Michelson," Glaucoma risk index: Automated glaucoma detection from color fundus images".

[4] R. Bock et al. / Medical Image Analysis 14, pp.471-481, 2010.

[5] D. EvangelinHepsiba, Josephine PusphaArasi," An Enhanced Automated Diagnosis Method for Glaucoma Detection Using Wavelet", IOSR Journal of Electronics and Communication Engineering (IOSR_JECE), ISSN: 2278_8735.Volume 9, Issue 3, pp.20_22, May_ Jun 2014 .

[6] L'aszl', G. Ny'ul," Retinal image analysis for automated glaucoma risk evaluation", Proc. of SPIE, Vol.7497_74971, pp.1_9.

[7] Preeti, JyotikaPruthi," Review of Image Processing Techniquefor Glaucoma Detection", International Journal of Computer Science and Mobile Computing, ISSN 2320-088X,Vol. 2, Issue. 11, pp. $99-105$, November 2013.

[8] K. Narasimhan, Vijayarekha, JogiNarayana, SivaPrasad and V. SatishKumar," Glaucoma Detection from Fundus Image Using Opencv", Research Journal of Applied Sciences, Engineering and Technology, ISSN: 2040_7467, Vol.4, No.24, pp.5459_5463, December 2012.

[9] HusandeepKaur, AmandeepKaur, " Early Stage Glaucoma Detection in Diabetic Patients: A Review", International Journal of Advanced Research in Computer Science and Software Engineering, ISSN: 2277 128X ,Volume 4, Issue 5,pp. 271_274 ,May 2014.

[10] Maryam Vatanparast, AhadHarati,"A Feasibilty Study on Detection of Neovascularization in Retinal Color images Using Texture".
[11] Nesreen et.al Eran.A.Edirisinghe,"Quantification of Corneal Neovascularization via Contourlet Transform based Segmentation of Blood Vessels", Ubiquitous Computing and Communication Journal, ISSN:1992_8424, Volume 7, pp. 1297_ 1308.

[12] Michael Feehan, John Hartman, Richard Durante1, Margaux A Morrison, Joan W Miller, Ivana K Kim and Margaret M DeAngelis," Identifying subtypes of patients with neovascularage_related macular degeneration by genotypic and cardiovascular risk characteristics", Feehan et al. BMC Medical Genetics,Vol.12,No.83,pp.1_8,2011.

[13] Krysten M Farjo, Jian_xing Ma," The potential of nanomedicine therapies to treat neovascular disease in the retina",Journal of angiogenesis research, Vol.2, No.21, pp.1_14.

[14] Chia_Ling,Yi_Lun, Chen,Kai_ShungLin,Chih_Hao Chan,,"Automatic Characterization of Classic Choroidal Neovascularization by Using AdaBoost for Supervised Learning”, IOVS, Vol. 52, No. 5,pp.2767_2773, April 2011.

[15] Muhammad WaseemKhan,'Diabetic Retinopathy Detection using Image Processing: A Survey", International Journal Of Emerging Technology \& Research,ISSN: 2347_6079, Volume 1, Issue 1,pp.16_20,Nov_Dec, 2013 .

[16] Roxan and Ronald H Silverman,"Anterior_segment imaging for assessment of glaucoma",Expert Rev. Ophthalmol,ISSN: 1746_9899,Vol.5,No.1, pp.5974,2010 .

[17] Jack Lee, Benny Chung Ying Zee, Qing Li, ” Detection of Neovascularization Based on Fractal and Texture Analysis with Interaction Effects in Diabetic Retinopathy",www.plosone.org, Vol.8,Issue.12, e75699, pp.1_9, 1 December 2013.

[18] L. T. Col Avinash Mishra, SaurabLuthra, Col V.K. Baranwal, Brig J.K.S. Parihar, SM, VSM, " An interesting case of rubeosisiridis with neovascular glaucoma in a young patient", medical journal armed forces india 69, pp.187_189,2013.

[19] DharmannaLamani, Dr. T. C. Manjunath, Dr. U P Kulkarni," Automated Detection of Neovascular Glaucoma through Fractal Dimension Method", International Journal of Computer Science and Information Technologies (IJCSIT), ISSN:0975_9646,Vol. 5 (4),pp.5252_5257, 2014.

[20] S.MuthuLakshmi,"Supervised Blood Vessel Segmentation in Retinal Images Using Feature Based Classification", International Journal of Advanced and Innovative Research, ISSN: 2278_7844, Volume 1, Issue 1,pp.1_5, June 2012. 